\title{
Modeling and Controlling a Robotic Convoy Using Guidance Laws Strategies
}

\author{
Fethi Belkhouche, Member, IEEE, and Boumediene Belkhouche, Member, IEEE
}

\begin{abstract}
This paper deals with the problem of modeling and controlling a robotic convoy. Guidance laws techniques are used to provide a mathematical formulation of the problem. The guidance laws used for this purpose are the velocity pursuit, the deviated pursuit, and the proportional navigation. The velocity pursuit equations model the robot's path under various sensors based control laws. A systematic study of the tracking problem based on this technique is undertaken. These guidance laws are applied to derive decentralized control laws for the angular and linear velocities. For the angular velocity, the control law is directly derived from the guidance laws after considering the relative kinematics equations between successive robots. The second control law maintains the distance between successive robots constant by controlling the linear velocity. This control law is derived by considering the kinematics equations between successive robots under the considered guidance law. Properties of the method are discussed and proven. Simulation results confirm the validity of our approach, as well as the validity of the properties of the method.
\end{abstract}

Index Terms-Guidance laws, relative kinematics equations, robotic convoy, tracking.

\section{INTRODUCTION}

$\mathbf{T}$ HE USE OF mobile robots to follow moving objects is a significant research problem in mobile robotics. This problem is addressed from different points of view (e.g., artificial vision, control theory, artificial intelligence), and various algorithms were suggested to achieve this task. Detection, tracking, pursuit, car-following, and autonomous driving are the major related themes. Most of the techniques used for moving objects following and pursuit can be divided into two main classes: model-based and feature-based. Visual servoing, which consists of controlling the motion of the robot based on visual features is among the most used techniques for tracking moving objects ([1]-[7]). Detection and tracking of humans using mobile robots is considered in ([1], [2]). In both papers, the tracking algorithm is based on artificial vision. In [3], another strategy based on visual servoing was used for tracking humans as well as rigid bodies. Different aspects in visual servoing such as robust real-time implementation [4], compensation of abrupt changes in the target motion [5], and camera uncertainties [6] were also addressed. Positioning a robot with respect to its target based on visual servoing was considered in [7]. Simulation of the pursuit of moving objects using a mobile robot and an artificial vision algorithm is considered in [8].

Manuscript received May 16, 2004; revised October 1, 2004. This paper was recommended by Associate Editor D. Y. Lee.

The authors are with the Electrical Engineering and Computer Science Department, Tulane University, New Orleans, LA 70118 USA (e-mail: belkhouf@eecs.tulane.edu).

Digital Object Identifier 10.1109/TSMCB.2005.846646
Moving potential field method [9] and Lyapunov theory [10] are also used for this purpose.

Car-following and autonomous convoy driving are quite similar problems [11]. These two problems are among the most important topics discussed within the intelligent transportation community. In general, the control problem of car-following can be divided into two problems.

1) Assist the driver of the following car by designing a control law for the speed in order to keep a safe constant distance from the lead vehicle. This is known as an adaptive cruise control (ACC) system.

2) The following car is fully autonomous. Thus, two control laws for the linear and angular velocities are considered.

Convoy driving can be seen as a generalization of car-following, where several vehicles follow each other. In fact convoy driving involves a cooperation task between robots. Cooperation between robots for group formation was considered in [12] and [13], where distributed and time-varying feedback controls were suggested. The problem of convoy driving can be seen as a special case of group formation. Military applications of convoy driving are the most obvious, where a given number of autonomous vehicles follow each other while keeping a safe constant distance. Other applications can be found, for example, in flexible factories, where an automated robotic convoy is used for product transportation. An algorithm for car convoy driving for military applications is suggested in [14]. The algorithm is based on fuzzy logic. A fuzzy logic controller is also suggested for driver assistance in a car-following problem [15]. The problem of robotic convoy from a communication point of view is considered in [16], where collaboration in terms of communication between robots in the convoy was considered. The same problem is considered in [17], but from a different point of view, where nonlinear control theory is used to design a feedback control law for the convoy control. Results concerning the control algorithm are rigorously proven. Another algorithm for robotic convoy driving based on vision is suggested in [18]. Similar to the case of following objects by robots, algorithms based on artificial vision are widely used in car-following problems ([19]-[21]). However, these control algorithms suffer from the following problems:

1) The control algorithm has to process in real time a huge flow of data coming from the camera. This task may be difficult, especially for fast tracking problems. Thus, the maximum computational power for image processing is an important issue.

2) The target (or the lead car) is detected only when it appears in the camera's field of view. Thus, the target must 
stay in the camera scope of the pursuer. This requirement is necessary to implement a vision-based algorithm [8].

Our aim here is to give a mathematical formulation of the problem of modeling and controlling a convoy of wheeled mobile robots. We propose a novel approach based on guidance laws strategies, where the robotic convoy is modeled in terms of the relative velocities of each lead robot with respect to its following robot. This approach is different from the other suggested approaches (e.g., artificial vision, nonlinear and fuzzy control). It results in important simplifications to the sensory system as compared to artificial vision algorithms. Moreover, our approach devises a systematic study of the problem based on kinematics equations. From such study, results concerning the tracking problem are rigorously proven. The guidance laws used for this purpose are the velocity pursuit, the deviated pursuit, and the proportional navigation. The velocity and deviated pursuits are special cases of the proportional navigation, which is the most known and the most used guidance law in practice. Some animals (e.g., dogs) use the velocity pursuit to catch moving objects [22]. Humans also may use some kinds of guidance laws in different sports, such as baseball [23].

In [24], the author discusses the answer to the question: "why the ants trails look so straight and nice". He establishes a mathematical model, which states that ants keep their trails straight and nice because they are navigating using the velocity pursuit guidance law. Insect and animal navigation continues to fascinate researchers in robotics, prompting the development of various ant-inspired, as well as other insect and animal-inspired, navigation algorithms ([25]-[29]).

An application of guidance laws to solve robotics problems can be found in [30], where the authors suggest an algorithm for robotic manipulator grasping of moving objects using the proportional navigation. A more recent and more detailed study for robotic catching of moving objects using the proportional navigation and its variants is discussed in ([31] and [32]). To the best of our knowledge, we are the first to use guidance laws to formulate the problem of modeling or controlling a robotic convoy. For the control of the convoy, we design two control laws for all following robots. The first control law is related to the angular velocity. The design of this law is based on guidance laws strategies. The second control law concerns the linear velocity, where the aim is to keep a constant distance between robots. This control law is derived using the kinematics equations under the guidance law between two successive robots. Both control laws are decentralized.

This paper is organized as follows. After describing the model for the robots and deriving the kinematics equations, we discus the tracking problem under the velocity pursuit, the deviated pursuit, and the proportional navigation. The control law for the angular velocity is then derived. Thereafter, a second control law for the linear velocity is derived for the three approaches, and important properties of the method are discussed and proven. Finally, simulation under various conditions is elaborated.

\section{Problem Formulation}

Given $N$ unconnected wheeled mobile robots which are expected to move in a convoy, the aim is to design a closed-loop

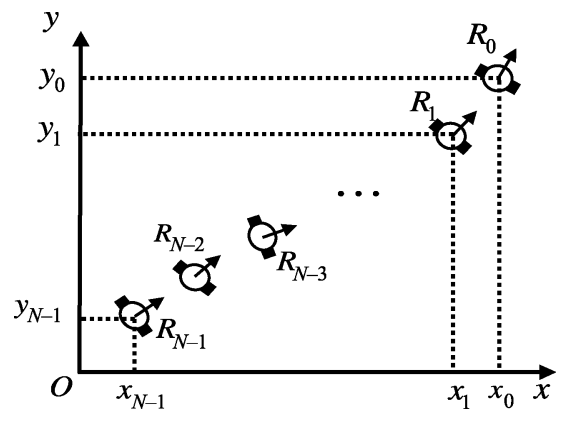

Fig. 1. Illustration of the robotic convoy.

control law for $N-1$ autonomous robots in order to follow the lead robot while keeping a constant distance from each other. The problem is essentially a motion matching problem. Fig. 1 shows the robotic convoy in the Cartesian frame of reference. The lead robot denoted by $R_{0}$ may be autonomous or may be remotely controlled. Let $P_{0}(t)=\left(x_{0}(t), y_{0}(t)\right)$ be the path traveled by the lead robot, with $\left(x_{0}(t), y_{0}(t)\right)$ being the coordinates of the lead robot in the Cartesian frame of reference. It is assumed that $P_{0}(t)=\left(x_{0}(t), y_{0}(t)\right)$ is a smooth function. We have the following assumptions.

1) The robots move in the horizontal plane.

2) Initially all the robots are in a position of a convoy.

3) The path of the lead robot $R_{0}$ is not known a priori.

4) For the implementation of the algorithm, it is assumed that each following robot has a sensory system capable of measuring the linear and angular velocities of its lead robot.

Note that this problem is a real-time problem, and therefore, the control strategy must be elaborated in real time. As we mentioned previously, our aim is to formulate the problem of robotic convoy mathematically based on the kinematics equations and suggest a solution based on guidance laws.

\section{Robots' Model and Relative Kinematics Equations}

We assume that all robots are modeled as wheeled mobile robots of the unicycle type. The $i^{\text {th }}$ robot is denoted by $R_{i}$, for $i=0, \ldots, N-1$. Robot $R_{i}$ has the following kinematics equations:

$$
\begin{aligned}
\dot{x}_{i} & =v_{i} \cos \theta_{i} \\
\dot{y}_{i} & =v_{i} \sin \theta_{i} \\
\dot{\theta}_{i} & =\omega_{i}
\end{aligned}
$$

where $\left(x_{i}, y_{i}\right)$ are the coordinates of the reference point of robot $R_{i}$ in the Cartesian frame of reference. $\theta_{i}$ is its orientation angle with respect to the positive x-axis. $v_{i}$ and $\omega_{i}$ are the linear and angular velocities, respectively. They are also the control inputs. Robot $R_{i}$ has the following linear velocity vector:

$$
\mathbf{v}_{i}=\dot{x}_{i} \mathbf{u}_{x}+\dot{y}_{i} \mathbf{u}_{y}
$$

where $\mathbf{u}_{x}$ and $\mathbf{u}_{y}$ are the unit vectors and $i=0, \ldots, N-1$. The relative range between robots $R_{i+1}$ and $R_{i}$ is denoted by $\mathbf{r}_{i, i+1}$ and is given by

$$
\mathbf{r}_{i, i+1}=\mathbf{r}_{i}-\mathbf{r}_{i+1}
$$




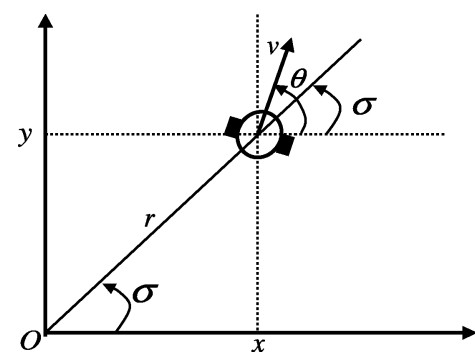

Fig. 2. Illustration of the Cartesian and polar coordinates.

which can be written as follows:

$$
\mathbf{r}_{i, i+1}=\left(x_{i}-x_{i+1}\right) \mathbf{u}_{x}+\left(y_{i}-y_{i+1}\right) \mathbf{u}_{y}
$$

with $i=0, \ldots, N-2$ in this case. By taking the time derivative of the relative range, we get the relative velocity

$$
\mathbf{v}_{i, i+1}=\mathbf{v}_{i}-\mathbf{v}_{i+1}
$$

which can be decomposed into two components with respect to the $\mathrm{x}$ - and $\mathrm{y}$ - axes as follows:

$$
\begin{aligned}
& \mathbf{v}_{(i, i+1) x}=v_{(i, i+1) x} \mathbf{u}_{x}=\left(\dot{x}_{i}-\dot{x}_{i+1}\right) \mathbf{u}_{x} \\
& \mathbf{v}_{(i, i+1) y}=v_{(i, i+1) y} \mathbf{u}_{y}=\left(\dot{y}_{i}-\dot{y}_{i+1}\right) \mathbf{u}_{y} .
\end{aligned}
$$

By considering the kinematics equations for the robots, we get

$$
\begin{aligned}
& v_{(i, i+1) x}=v_{i} \cos \theta_{i}-v_{i+1} \cos \theta_{i+1} \\
& v_{(i, i+1) y}=v_{i} \sin \theta_{i}-v_{i+1} \sin \theta_{i+1} .
\end{aligned}
$$

This model represents the relative velocities of the robot $R_{i}$ with respect to the robot $R_{i+1}$ in the Cartesian frame of reference.

In this paper, we use polar coordinates representation for all robots. Polar coordinates are used by several authors ([33], [34]) to design different control laws for robots of the unicycle type. Fig. 2 illustrates the Cartesian and polar coordinates representations. Consider the following change of variable:

$$
\begin{aligned}
& x=r \cos \sigma \\
& y=r \sin \sigma
\end{aligned}
$$

where $r$ is the radial coordinate and $\sigma$ is the angular coordinate. Under this change of variable, the kinematics model for the robots is

$$
\begin{aligned}
\dot{r} & =v \cos (\theta-\sigma) \\
r \dot{\sigma} & =v \sin (\theta-\sigma) .
\end{aligned}
$$

This system is obtained by noting that

$$
\dot{r}=\frac{\dot{x} x+\dot{y} y}{r}
$$

and

$$
\dot{\sigma}=\frac{\dot{y} x-y \dot{x}}{r^{2}}
$$

and using system (8) with the kinematics equations of the robots. Before we discuss the control strategies, we need to introduce some definitions related to geometric quantities,

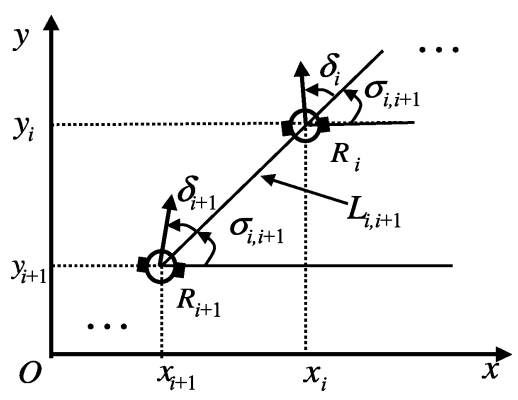

Fig. 3. Geometry of the tracking problem.

since guidance laws are based on geometry. The geometry of the tracking problem is illustrated in Fig. 3. The line of sight between robot $R_{i+1}$ and robot $R_{i}$ is the virtual straight line starting from the reference point of robot $R_{i+1}$ and directed toward robot $R_{i}$. This line is denoted by $L_{i, i+1}$. The line of sight angle is the angle between the positive $\mathrm{x}$-axis and the line of sight. For the line of sight $L_{i, i+1}$ between robots $R_{i+1}$ and $R_{i}$, we have the line of sight angle $\sigma_{i, i+1}$. The relative distance between robots $R_{i+1}$ and $R_{i}$ is given by

$$
r_{i, i+1}=\sqrt{\left(x_{i}-x_{i+1}\right)^{2}+\left(y_{i}-y_{i+1}\right)^{2}}
$$

and the line of sight angle is given by

$$
\tan \sigma_{i, i+1}=\frac{y_{i}-y_{i+1}}{x_{i}-x_{i+1}}
$$

For a convoy of $N$ robots, we have $N-1$ lines of sight and $N-1$ line of sight angles. Consider the relative velocity between two successive robots

$$
\mathbf{v}_{i, i+1}=\mathbf{v}_{i}-\mathbf{v}_{i+1} .
$$

$\mathbf{v}_{i, i+1}$ can be decomposed into two components along and across the line of sight $L_{i, i+1}$. The radial velocity denoted by $\mathbf{v}_{(i, i+1) R}$ is the component of $\mathbf{v}_{i, i+1}$ along the line of sight; the tangential velocity denoted by $\mathbf{v}_{(i, i+1) T}$ is the component of $\mathbf{v}_{i, i+1}$ across the line of sight. The radial velocity and the tangential velocity are respectively expressed as

$$
\begin{aligned}
& v_{(i, i+1) R}=\dot{r}_{i, i+1}=v_{i} \cos \delta_{i}-v_{i+1} \cos \delta_{i+1} \\
& v_{(i, i+1) T}=r_{i, i+1} \dot{\sigma}_{i, i+1}=v_{i} \sin \delta_{i}-v_{i+1} \sin \delta_{i+1} .
\end{aligned}
$$

Equations (15) and (16) provide a kinematics model for the robotic convoy. They represent the relative velocities of the lead robot $R_{i}$ with respect to the following robot $R_{i+1}$, along the line of sight $L_{i, i+1}$, and normal to the line of sight, respectively. The angle $\delta$ is a lead/follow angle depending on the role of the robot being in a lead or following position. It represents the angle between the line of sight and the velocity vector. For each pair of lead-following robots $\left(R_{i}, R_{i+1}\right)$ joined by the line of sight $L_{i, i+1}$, the lead/follow angles are, respectively, expressed as

$$
\begin{aligned}
\delta_{i} & =\theta_{i}-\sigma_{i, i+1} \\
\delta_{i+1} & =\theta_{i+1}-\sigma_{i, i+1} .
\end{aligned}
$$

Clearly, $R_{N-1}$ is excluded in (17) and $R_{0}$ is excluded in (18). Thus, the lead/follow angle is a function of the robot's orientation angle and the line of sight angle. 


\section{TRACKING PROBLEM}

Our aim is to design a decentralized closed-loop control law for the angular velocity of $N-1$ robots. The control law allows each robot, except $R_{0}$, to follow its leading robot (except $R_{N-1}$ ). Our strategy is based on the pursuit and the proportional navigation guidance laws, which are closed-loop control laws. The pursuit and the proportional navigation are among the most important guidance laws ([22], [35], [36]) (initially designed for missile guidance). The pursuit is a special case of the proportional navigation guidance law, which is the most known and the most used guidance law in practice. There exist two variants of the pursuit: velocity pursuit and deviated pursuit. The two variants are discussed and used in this paper. For the proportional navigation, we use specific values of the proportionality factor in such a way that the proportional navigation appears as a deviated pursuit with time-varying deviation angle.

\section{A. Principle of the Guidance Laws}

The guidance laws used here are based on the geometry and the kinematics equations. The principle of the velocity pursuit is to make the velocity vector of the pursuer lying on the line of sight joining the pursuer and its target point. Different sensorbased control systems can be built on this principle. For the deviated pursuit, there exists a nonzero angle between the line of sight and the velocity vector. Even though the pursuit seems to be an easy problem because of the simplicity of its principle, its equations are rather difficult to solve [24]. The proportional navigation can be seen as a generalization of the pursuit. For the proportional navigation, the angular velocity of the pursuer is proportional to the rate of turn of the line of sight angle. These guidance laws will be discussed in more details when elaborating the corresponding control law for the robotic convoy.

\section{B. Robotic Convoy Based on the Velocity Pursuit Guidance Law}

In the velocity pursuit, the velocity vector of robot $R_{i+1}$ lies on the line of sight joining robot $R_{i+1}$ and robot $R_{i}$. This means that the velocity vector of $R_{i+1}$ lies in the line of sight $L_{i, i+1}$. The velocity pursuit is characterized by two relations, one vectorial and one scalar. These relations are

$$
\mathbf{v}_{i+1} \times \mathbf{r}_{i, i+1}=\mathbf{0}
$$

This relation states that $v_{i+1}$ lies on $L_{i, i+1}$. The scalar relation concerns the direction and is stated as follows:

$$
\mathbf{v}_{i+1} \cdot \mathbf{r}_{i, i+1}>0
$$

Under (19) and (20), the orientation angle of $R_{i+1}$ is given by

$$
\theta_{i+1}=\sigma_{i, i+1}
$$

By taking the derivative in (21), we get

$$
\omega_{i+1}=\dot{\sigma}_{i, i+1}
$$

which means that the angular velocity of $R_{i+1}$ is equal to the rate of turn of the line of sight angle between robots $R_{i+1}$ and
$R_{i}$. The values for the radial and tangential velocity components under the velocity pursuit are given by

$$
\begin{aligned}
\dot{r}_{i, i+1} & =v_{i} \cos \delta_{i}-v_{i+1} \\
r_{i, i+1} \dot{\sigma}_{i, i+1} & =v_{i} \sin \delta_{i}
\end{aligned}
$$

which can be written as

$$
\begin{aligned}
\dot{r}_{i, i+1} & =v_{i} \cos \left(\theta_{i}-\sigma_{i, i+1}\right)-v_{i+1} \\
r_{i, i+1} \dot{\sigma}_{i, i+1} & =v_{i} \sin \left(\theta_{i}-\sigma_{i, i+1}\right)
\end{aligned}
$$

This is a simple two-dimensional nonlinear system. However, the analytical solution for this system is not possible in general. The kinematics equations for the motion of robot $R_{i+1}(i=$ $0, \ldots, N-2)$ under the velocity pursuit are given by

$$
\begin{aligned}
\dot{x}_{i+1} & =v_{i+1} \cos \sigma_{i, i+1} \\
\dot{y}_{i+1} & =v_{i+1} \sin \sigma_{i, i+1} \\
\dot{\theta}_{i+1} & =\omega_{i+1}=\dot{\sigma}_{i, i+1} .
\end{aligned}
$$

\section{Robotic Convoy Based on the Deviated Pursuit Guidance Law}

In the deviated pursuit, there exists a constant nonzero angle between the velocity vector of robot $R_{i+1}$ and the line of sight $L_{i, i+1}$. In this case, (21) becomes

$$
\alpha_{i+1}=\theta_{i+1}-\sigma_{i, i+1}
$$

where $\alpha_{i+1}$ is a constant angle for all following robots. Equation (22) is also valid for the case of the deviated pursuit. The sign of the angle $\alpha_{i+1}$ and the direction of $R_{i}$ determine whether it is lead or lag pursuit.

In the case of the deviated pursuit, the radial and tangential velocities between two successive robots are

$$
\begin{aligned}
\dot{r}_{i, i+1} & =v_{i} \cos \delta_{i}-v_{i+1} \cos \alpha_{i+1} \\
r_{i, i+1} \dot{\sigma}_{i, i+1} & =v_{i} \sin \delta_{i}-v_{i+1} \sin \alpha_{i+1}
\end{aligned}
$$

There exists a constraint on the angles $\alpha_{i+1}$, where $\alpha_{i+1} \in$ $(-(\pi / 2), \pi / 2)$. Otherwise, it becomes an escape instead of a pursuit. Even though the tracking is accomplished successfully when $\alpha_{i+1} \in(-(\pi / 2), \pi / 2)$, the error in the path between two successive robots becomes important as $\alpha_{i+1} \rightarrow \pm(\pi / 2)$, which is not convenient for robotic convoy or car-following problems. For robotic convoy, the aim of the following robots is to track the same trajectory as the lead robot. To accomplish this task, small values of $\alpha_{i+1}$ are recommended, since they represent small deviations from the line of sight angle. Consequently, the deviated pursuit can be used in a pursuit fashion. Under these conditions, there exists a maximum tolerable value for $\alpha_{i+1}$, which we denote by $\alpha_{T}$. $\alpha_{T}$ can be chosen depending on the application and also the type of trajectory traveled by the convoy. In this paper we set $\alpha_{T}=10^{\circ}$, restricting the interval for $\alpha_{i+1}$ such that $\alpha_{i+1} \in\left[-10^{\circ}, 10^{\circ}\right.$. It is worth noting that $\alpha_{i+1}$ can be used in some situations for path correction, as we 
will see in the simulation. The kinematics equations for the motion of robot $R_{i+1}(i=0, \ldots, N-2)$ under the deviated pursuit are

$$
\begin{aligned}
\dot{x}_{i+1} & =v_{i+1} \cos \left(\alpha_{i+1}+\sigma_{i, i+1}\right) \\
\dot{y}_{i+1} & =v_{i+1} \sin \left(\alpha_{i+1}+\sigma_{i, i+1}\right) \\
\dot{\theta}_{i+1} & =\omega_{i+1}=\dot{\sigma}_{i, i+1} .
\end{aligned}
$$

This case can be seen as the general case of the pursuit, since the velocity pursuit can be recovered when $\alpha_{i+1}=0$.

\section{Robotic Convoy Based on the Proportional Navigation Guidance Law}

As mentioned previously, the velocity and deviated pursuits are special cases of the proportional navigation guidance law. In the proportional navigation, the following relation is satisfied:

$$
\omega_{i+1}=K \dot{\sigma}_{i, i+1} .
$$

The velocity and deviated pursuit are recovered for $K=1$. For the velocity pursuit, the initial state satisfies

$$
\theta_{i+1}\left(t_{0}\right)=\sigma_{i, i+1}\left(t_{0}\right)
$$

Usually for the proportional navigation, $K$ is a constant real number with $K>1$. For higher values of $K$, the tracking problem under the proportional navigation becomes a rendezvous problem. In order to use the proportional navigation guidance law for the convoy problem, we suggest that

$$
1<K \leq 1+\eta
$$

where $\eta$ is a small real number describing how much the proportional navigation will be deviated from the velocity pursuit. Similar to the deviated pursuit, there exists a maximum tolerable value for $\eta$, which is denoted by $\eta_{\max } \cdot \eta_{\max }$ can be chosen depending on the application and the trajectory type of the convoy. We suggest in this paper to take $\eta_{\max }=0.1$, which means that the proportional navigation will be deviated from the velocity pursuit by $10 \%$ of the value of the line of sight angle. Thus the proportional navigation will be used in a similar way to the deviated pursuit, but with a time-varying deviation angle. For the purpose of robotic convoy, we define the proportional navigation by the following system:

$$
\begin{aligned}
\theta_{i+1} & =K \sigma_{i, i+1} \\
1 & <K \leq 1.1 .
\end{aligned}
$$

The kinematics equations under the proportional navigation between two successive robots are the following:

$$
\begin{aligned}
\dot{r}_{i, i+1} & =v_{i} \cos \delta_{i}-v_{i+1} \cos \left(M \sigma_{i, i+1}\right) \\
r_{i, i+1} \dot{\sigma}_{i, i+1} & =v_{i} \sin \delta_{i}-v_{i+1} \sin \left(M \sigma_{i, i+1}\right)
\end{aligned}
$$

with $M=K-1$. These equations are similar to the equations of the deviated pursuit. The main difference is that the deviation angle here is proportional to the line of sight angle, while it is constant for the deviated pursuit. The kinematics equations for the motion of robot $R_{i+1}(i=0, \ldots, N-2)$ under the proportional navigation are given by

$$
\begin{aligned}
\dot{x}_{i+1} & =v_{i+1} \cos \left(K \sigma_{i, i+1}\right) \\
\dot{y}_{i+1} & =v_{i+1} \sin \left(K \sigma_{i, i+1}\right) \\
\dot{\theta}_{i+1} & =\omega_{i+1}=K \dot{\sigma}_{i, i+1} .
\end{aligned}
$$

Clearly, the case when $K=1$ corresponds to the velocity pursuit.

In the next section, we address the problem of keeping the distance between robots constant. Also, important properties of the control laws are discussed and proven.

\section{Convoy With Constant Distance Between Robots}

The aim here is to design a second control law to keep the relative distance between pairs of robots $\left(R_{0}, R_{1}\right),\left(R_{1}, R_{2}\right), \ldots,\left(R_{N-2}, R_{N-1}\right)$ constant for all three control strategies. The control input is the linear velocity for $(N-1)$ robots. For all three strategies, the derivation of the second control law is simple and based on the kinematics equations between two successive robots. We also elaborate an analysis of the strategies being used, and prove some important results concerning the tracking problem in the convoy.

\section{A. Velocity Pursuit With Constant Distance Between Robots}

We rewrite the equation for the relative radial velocity under the velocity pursuit guidance law as

$$
\dot{r}_{i, i+1}=v_{i} \cos \delta_{i}-v_{i+1} .
$$

The relative distance between $R_{i+1}$ and $R_{i}$ can be decreasing, constant, or increasing depending on the values of $v_{i+1}, v_{i}$ and $\delta_{i}$ (which is a function of the orientation angle of robot $R_{i}$ and the line of sight angle between robots $R_{i+1}$ and $R_{i}$ ). If $\dot{r}_{i, i+1}<$ 0 meaning that $v_{i} \cos \delta_{i}<v_{i+1}$, then the relative distance is decreasing and $R_{i+1}$ will collide with $R_{i}$. If $\dot{r}_{i, i+1}>0$ meaning that $v_{i} \cos \delta_{i}>v_{i+1}$, then the relative distance is increasing and $R_{i}$ will escape from $R_{i+1}$. The third case which is desired is characterized by $\dot{r}_{i, i+1}=0$. In this case, the relative distance is constant. From (35), we have

$$
v_{i+1}=v_{i} \cos \delta_{i}
$$

which is equivalent to

$$
v_{i+1}=v_{i} \cos \left(\theta_{i}-\sigma_{i, i+1}\right) .
$$

By replacing $\sigma_{i, i+1}$ by its value under the velocity pursuit, (37) can be written as

$$
v_{i+1}=v_{i} \cos \left(\theta_{i}-\theta_{i+1}\right) .
$$

Equation (37) describes the closed-loop control strategy for the linear velocity of robot $R_{i+1}$ in order to keep constant distance from robot $R_{i}$. Since the relative distance $r_{i, i+1}$ between two successive robots is constant, we will denote it by

$$
r_{i, i+1}=d_{i 0}, \quad i=0, \ldots, N-2 .
$$


In the case of the velocity pursuit, it is possible to write the kinematics equations of $R_{i+1}$ as a function of the position of $R_{i}$ in the Cartesian coordinates as follows:

$$
\begin{aligned}
\dot{x}_{i+1} & =\frac{v_{i+1}}{d_{i 0}}\left(x_{i}-x_{i+1}\right) \\
\dot{y}_{i+1} & =\frac{v_{i+1}}{d_{i 0}}\left(y_{i}-y_{i+1}\right) \\
\dot{\theta}_{i+1} & =\dot{\sigma}_{i, i+1} .
\end{aligned}
$$

This system can be obtained by replacing $\cos \sigma_{i, i+1}$ and $\sin \sigma_{i, i+1}$ by their values

$$
\begin{aligned}
\cos \sigma_{i, i+1} & =\frac{x_{i}-x_{i+1}}{d_{i 0}} \\
\sin \sigma_{i, i+1} & =\frac{y_{i}-y_{i+1}}{d_{i 0}} .
\end{aligned}
$$

From the first two equations in (40), the equation for the path can be written as a linear differential equation

$$
\dot{p}_{i+1}=\frac{v_{i+1}}{d_{i 0}}\left(p_{i}-p_{i+1}\right) \text {. }
$$

The path of $R_{i+1}$ can be obtained from the solution of (42) when the path of $R_{i}$ is known. Equation (42) has the general form of a nonautonomous linear scalar differential equation with single input. Since the equation is linear, the closed-form solution can be formulated as follows:

$$
\begin{aligned}
p_{i+1}(t)=e^{\int_{t_{0}}^{t} a(u) d u} & p_{i+1}\left(t_{0}\right)+e^{\int_{t_{0}}^{t} a(u) d u} \\
& \times \int_{t_{0}}^{t} \frac{v_{i+1}(s)}{d_{i 0}} p_{i}(s) e^{-\int_{t_{0}}^{s} a(u) d u} d s
\end{aligned}
$$

with $a=-v_{i+1} / d_{i 0}, a$ is time-varying when $v_{i+1}$ is timevarying, and $a<0$. When $v_{i+1}$ is constant, we get

$p_{i+1}(t)=e^{a\left(t-t_{0}\right)} p_{i+1}\left(t_{0}\right)+e^{a\left(t-t_{0}\right)} \frac{v_{i+1}}{d_{i 0}} \int_{t_{0}}^{t} e^{-a\left(s-t_{0}\right)} p_{i}(s) d s$.

Thus, the path of robot $R_{i+1}$ can be obtained from robot $R_{i}$, and the path of all robots can be obtained from the path of robot $R_{0}$.

The equations obtained here for the velocity pursuit can be used to model the problem of tracking for various sensor-based control laws, for example, when the control law is based on artificial vision or light sources. This renders the mathematical analysis of these control algorithms possible.

As shown in (22), the angular velocity in the pursuit law is equal to the rate of turn of the line of sight angle between two successive robots. Thus $\sigma_{i, i+1}$ must be a bounded function. This point is captured by the following proposition.

Proposition 1: Under the velocity pursuit with constant distance between two successive robots, the control input for the angular velocity is a bounded function.

Proof: The proof is based on the use of the equation for the relative tangential velocity under the velocity pursuit, which is given by

$$
r_{i, i+1} \dot{\sigma}_{i, i+1}=v_{i} \sin \left(\theta_{i}-\sigma_{i, i+1}\right)
$$

and the control law for $\omega_{i+1}$, which is given by (22). Under the velocity pursuit with constant distance between two successive robots, the relative distance $r_{i, i+1}$ in the expression of the tangential velocity is constant and replaced by $d_{i 0}$. In this case, we get

$$
\omega_{i+1}=\frac{v_{i} \sin \left(\theta_{i}-\sigma_{i, i+1}\right)}{d_{i 0}} \leq\left|\frac{v_{i}}{d_{i 0}}\right| .
$$

Thus, $\omega_{i+1}$ is a bounded function.

In a convoy, when the robots are controlled based on the velocity pursuit law, the aim of robot $R_{i+1}$ is to imitate its lead robot $R_{i}$ in the motion. This is stated mathematically as follows.

Proposition 2: Under the velocity pursuit, the orientation angle of robot $R_{i+1}$ tracks the orientation angle of robot $R_{i}$, i.e., $\theta_{i+1}(t) \rightarrow \theta_{i}(t)$.

Proof: The proof is based on the use of the equation for the tangential velocity subject to the velocity pursuit strategy, which gives

$$
\dot{\theta}_{i+1}=\frac{v_{i}}{d_{i 0}} \sin \left(\theta_{i}-\theta_{i+1}\right)=f\left(\theta_{i+1}, t\right)
$$

This system has two equilibrium solutions, namely $\theta_{i+1}^{*}(t)=$ $\theta_{i}(t)$ and $\theta_{i+1}^{*}(t)=\theta_{i}(t)+\pi$. By linearizing near each equilibrium solution, we get

$$
\left.\frac{\partial f}{\partial \theta_{i+1}}\right|_{\theta_{i+1}^{*}=\theta_{i}}=-\frac{v_{i}}{d_{i 0}}<0
$$

and

$$
\left.\frac{\partial f}{\partial \theta_{i+1}}\right|_{\theta_{i+1}^{*}=\theta_{i}+\pi}=\frac{v_{i}}{d_{i 0}}>0 .
$$

Both equilibrium solutions are hyperbolic, which means that there exists an equivalence between the linear and the nonlinear systems. Thus $\theta_{i+1}^{*}(t)=\theta_{i}(t)$ is asymptotically stable, and $\theta_{i+1}^{*}(t)=\theta_{i}(t)+\pi$ is unstable. This means that $\theta_{i+1}(t)$ will track its stable equilibrium solution which is $\theta_{i}(t)$. Thus, $\theta_{i+1}(t) \rightarrow \theta_{i}(t)$.

Proposition 3: Under control laws (21) and (37), the linear velocity of robot $R_{i+1}$ tracks the linear velocity of robot $R_{i}$, i.e., $v_{i+1} \rightarrow v_{i}$.

Proof: By combining the previous result and the equation for the velocity of $R_{i+1}$ given by

$$
v_{i+1}=v_{i} \cos \left(\theta_{i}-\theta_{i+1}\right)
$$

we get $v_{i+1} \rightarrow v_{i}$ as $\theta_{i+1} \rightarrow \theta_{i}$.

An important property of the velocity pursuit is that $R_{i+1}$ aims to null the error in the orientation angle $e_{1}=\theta_{i+1}-\theta_{i}$ by nulling the error $e_{2}=\sigma_{i, i+1}-\theta_{i}$, therefore, in the velocity pursuit $\sigma_{i, i+1} \rightarrow \theta_{i}$.

\section{B. Deviated Pursuit With Constant Distance Between Robots}

The derivation of the second control law for $N-1$ robots in order to keep the distance between two successive robots constant is similar to the previous case. For robots navigating under the deviated pursuit, we have

$$
v_{i+1}=v_{i} \frac{\cos \left(\theta_{i}-\sigma_{i, i+1}\right)}{\cos \alpha_{i+1}}
$$

with $\alpha_{i+1} \in\left[-10^{\circ}, 10^{\circ}\right]$. Similar to the velocity pursuit, the control input for the angular velocity in the case of the deviated 
pursuit is also a bounded function. This can be proven using the equation for the line of sight angle rate.

Proposition 4: Under the deviated pursuit with constant distance between two successive robots, the control input for the angular velocity is a bounded function.

Proof: By replacing $v_{i+1}$ by its value, we have for the tangential relative velocity under the deviated pursuit with constant relative distance

$$
d_{i 0} \dot{\sigma}_{i, i+1}=v_{i}\left[\sin \delta_{i}-\frac{\cos \delta_{i}}{\cos \alpha_{i+1}} \sin \alpha_{i+1}\right] .
$$

Using trigonometric identities, we get

$$
d_{i 0} \dot{\sigma}_{i, i+1}=v_{i} \frac{\sin \left(\delta_{i}-\alpha_{i+1}\right)}{\cos \alpha_{i+1}}
$$

which is equivalent to

$$
\omega_{i+1}=\frac{v_{i}}{d_{i 0}} \frac{\sin \left(\delta_{i}-\alpha_{i+1}\right)}{\cos \alpha_{i+1}}
$$

(from the definition of the deviated pursuit). Thus

$$
\omega_{i+1} \leq\left|\frac{v_{i}}{d_{i 0} \cos \alpha_{i+1}}\right|
$$

Recall that $\alpha_{i+1}$ is constant.

Since $\alpha_{i+1} \in\left[-10^{\circ}, 10^{\circ}\right]$, the value of $\omega_{i+1}$ in this case is in general greater than the case of the velocity pursuit. It is the same for the linear velocity. Similar to the velocity pursuit, each following robot navigating under the deviated pursuit imitates its lead robot. This is stated as follows.

Proposition 5: Under the deviated pursuit, the orientation angle of $R_{i+1}$ tracks the orientation angle of $R_{i}$, i.e., $\theta_{i+1}(t) \rightarrow$ $\theta_{i}(t)$.

Proof: By considering (53) which gives the rate of the line of sight angle, and replacing $\delta_{i}$ by its value, we get

$$
d_{i 0} \dot{\sigma}_{i, i+1}=v_{i} \frac{\sin \left(\theta_{i}-\sigma_{i, i+1}-\alpha_{i+1}\right)}{\cos \alpha_{i+1}} .
$$

By replacing $\alpha_{i+1}$ by its value under the deviated pursuit (26) and $\dot{\sigma}_{i, i+1}$ by $\dot{\theta}_{i+1}$, we get

$$
d_{i 0} \dot{\theta}_{i+1}=v_{i} \frac{\sin \left(\theta_{i}-\theta_{i+1}\right)}{\cos \alpha_{i+1}} .
$$

This equation is similar to (47), with the same equilibrium solutions. The linearization near $\theta_{i+1}^{*}(t)=\theta_{i}(t)$ and $\theta_{i+1}^{*}(t)=$ $\theta_{i}(t)+\pi$ gives

$$
\left.\frac{\partial f}{\partial \theta_{i+1}}\right|_{\theta_{i+1}^{*}=\theta_{i}}=-\frac{v_{i}}{d_{i 0} \cos \alpha_{i+1}}<0
$$

and

$$
\left.\frac{\partial f}{\partial \theta_{i+1}}\right|_{\theta_{i+1}^{*}=\theta_{i}+\pi}=\frac{v_{i}}{d_{i 0} \cos \alpha_{i+1}}>0 .
$$

Since $\alpha_{i+1} \in\left[-10^{\circ}, 10^{\circ}\right], \cos \alpha_{i+1}>0$. Thus, $\theta_{i+1}^{*}(t)=\theta_{i}(t)$ is the asymptotically stable solution, and $\theta_{i+1}$ tracks its stable solution, i.e., $\theta_{i+1}(t) \rightarrow \theta_{i+1}^{*}(t)=\theta_{i}(t)$.
The following result relates the linear velocities.

Proposition 6: Under control laws (26) and (51), the linear velocity of $R_{i+1}$ tracks the linear velocity of $R_{i}$.

Proof: From (51), by replacing $\sigma_{i, i+1}$ by its value under the deviated pursuit, we get

$$
v_{i+1}=v_{i} \frac{\cos \left(\theta_{i}-\theta_{i+1}+\alpha_{i+1}\right)}{\cos \alpha_{i+1}} .
$$

As proven, we have under the deviated pursuit $\theta_{i+1}(t) \rightarrow \theta_{i}(t)$, from which we get $v_{i+1} \rightarrow v_{i}$.

As we mentioned previously, for the velocity pursuit, $R_{i+1}$ aims to null the difference $\sigma_{i, i+1}-\theta_{i}$. This is not the case for the deviated pursuit, where $R_{i+1}$ aims to null the error in the orientation angle by nulling the quantity $e=\sigma_{i, i+1}-\theta_{i}+\alpha_{i+1}$, therefore, $\sigma_{i, i+1} \rightarrow \theta_{i}-\alpha_{i+1}$ in the deviated pursuit.

\section{Proportional Navigation With Constant Distance Between Robots}

For robots navigating under the proportional navigation, constant relative distance between two successive robots requires

$$
v_{i+1}=v_{i} \frac{\cos \left(\theta_{i}-\sigma_{i, i+1}\right)}{\cos \left(M \sigma_{i, i+1}\right)}
$$

with $0<M \leq 0.1$. The following proposition states that the angular velocity under the proportional navigation guidance law is bounded.

Proposition 7: Under the proportional navigation with constant distance between two successive robots, the control input for the angular velocity is a bounded function.

Proof: For the proportional navigation with constant relative distance, the tangential velocity is given by

$$
d_{i 0} \dot{\sigma}_{i, i+1}=v_{i}\left[\sin \delta_{i}-\frac{\cos \delta_{i}}{\cos \left(M \sigma_{i, i+1}\right)} \sin \left(M \sigma_{i, i+1}\right)\right]
$$

which can be reduced using trigonometric identities to the following equation:

$$
d_{i 0} \dot{\sigma}_{i, i+1}=v_{i} \frac{\sin \left(\delta_{i}-M \sigma_{i, i+1}\right)}{\cos \left(M \sigma_{i, i+1}\right)}
$$

which is equivalent to

$$
\omega_{i+1}=K v_{i} \frac{\sin \left(\delta_{i}-M \sigma_{i, i+1}\right)}{d_{i 0} \cos \left(M \sigma_{i, i+1}\right)} .
$$

Since $0<M \leq 0.1$, the values of $\left|\cos \left(M \sigma_{i, i+1}\right)\right|$ will lie in a given interval $[\epsilon, 1]$, with $\epsilon$ near 1 (for $\sigma_{i, i+1}=90^{\circ}$, $\left.\cos \left(0.1 \sigma_{i, i+1}\right) \simeq 0.9877\right)$. Thus, $\omega_{i+1}$ is a bounded function in this case also.

Similar to the deviated pursuit, the value of $\omega_{i+1}$ in this case is in general greater than the case of the velocity pursuit. It is the same for the linear velocity. Similar to the pursuit, we have the following results for the proportional navigation.

Proposition 8: Under the proportional navigation with $1<$ $K \leq 1.1$, the orientation angle of $R_{i+1}$ tracks the orientation angle of $R_{i}$, i.e., $\theta_{i+1}(t) \rightarrow \theta_{i}(t)$. 
Proof: The proof is similar to the previous cases. From (63) after replacing $\delta_{i}$ by its value, we get:

$$
d_{i 0} \dot{\sigma}_{i, i+1}=v_{i} \frac{\sin \left(\theta_{i}-\sigma_{i, i+1}-M \sigma_{i, i+1}\right)}{\cos \left(M \sigma_{i, i+1}\right)} .
$$

By replacing $\sigma_{i, i+1}$ and $\dot{\sigma}_{i, i+1}$ by their values under the proportional navigation, we get

$$
\begin{aligned}
d_{i 0} \dot{\theta}_{i+1} & =K v_{i} \frac{\sin \left(\theta_{i}-\frac{\theta_{i+1}}{K}-\frac{M \theta_{i+1}}{K}\right)}{\cos \left(\frac{M \theta_{i+1}}{K}\right)} \\
& =K v_{i} \frac{\sin \left(\theta_{i}-\theta_{i+1}\right)}{\cos \left(\frac{M \theta_{i+1}}{K}\right)}
\end{aligned}
$$

(recall that $M+1=K$ ). So the equilibrium solutions for this system are similar to the previous cases. By using the same technique as before, it turns out that

$$
\left.\frac{\partial f}{\partial \theta_{i+1}}\right|_{\theta_{i+1}^{*}=\theta_{i}}=-\frac{K v_{i}}{d_{i 0} \cos \left(\frac{M \theta_{i}}{K}\right)}<0 .
$$

This quantity is negative. Since $0<(M / K) \leq 0.1$, we have $\cos \left(M \theta_{i} / K\right)>0, \forall \theta_{i}$ (under the transformation $0.1 \theta_{i}$, the interval $\left[90^{\circ}, 270^{\circ}\right]$ is mapped to $\left[9^{\circ}, 27^{\circ}\right]$, for which the cosine is positive). Thus, $\theta_{i+1}^{*}=\theta_{i}$ is an asymptotically stable solution, and the orientation angle of $R_{i+1}$ tracks the orientation angle of $R_{i}$, i.e., $\theta_{i+1}(t) \rightarrow \theta_{i}(t)$. Note that, similar to the previous cases, the other equilibrium solution $\theta_{i+1}^{*}=\theta_{i}+\pi$ is unstable.

The following proposition states that $R_{i+1}$ imitates $\mathrm{R}_{i}$ in terms of the linear velocity.

Proposition 9: Under control laws (32) and (61), the linear velocity of $R_{i+1}$ tracks the linear velocity of $R_{i}$.

Proof: From (61), replacing $\sigma_{i, i+1}$ by its value under the proportional navigation, we get

$$
v_{i+1}=v_{i} \frac{\cos \left(\theta_{i}-\frac{\theta_{i+1}}{K}\right)}{\cos \left(\frac{M \theta_{i+1}}{K}\right)} .
$$

Since $\theta_{i+1}(t) \rightarrow \theta_{i}(t)$ under the proportional navigation, when $\theta_{i+1}(t) \rightarrow \theta_{i}(t)$, we get

$$
\begin{aligned}
v_{i+1} & =v_{i} \frac{\cos \left(\theta_{i}-\frac{\theta_{i}}{K}\right)}{\cos \left(\frac{M \theta_{i}}{K}\right)} \\
& =v_{i} \frac{\cos \left(\frac{(K-1) \theta_{i}}{K}\right)}{\cos \left(\frac{M \theta_{i}}{K}\right)} .
\end{aligned}
$$

Recall that $M=K-1$. Thus, we get $v_{i+1} \rightarrow v_{i}$ as $\theta_{i+1}(t) \rightarrow$ $\theta_{i}(t)$.

Under the proportional navigation, $R_{i+1}$ aims to null the error in the orientation angle between successive robots by nulling the difference $\sigma_{i, i+1}-\left(\theta_{i} / K\right)$, thus $\sigma_{i, i+1} \rightarrow\left(\theta_{i} / K\right)$ in the proportional navigation.

Equations (37), (51), and (61) show that the linear velocity of robot $R_{i+1}$ is proportional to the velocity of robot $R_{i}$ with a time-varying proportionality factor

$$
v_{i+1}=k(t) v_{i}
$$

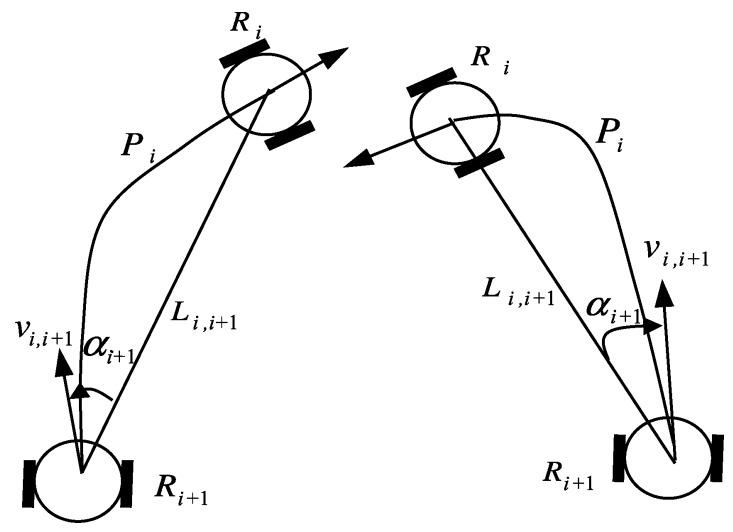

Fig. 4. Illustration of the path correction using the deviated pursuit.

Clearly, the linear velocity of robot $R_{i+1}$ depends on the following factors.

1) The linear and angular velocity of robot $R_{i}$.

2) The line of sight angle between robots $R_{i+1}$ and $R_{i}$, which is a geometrical quantity.

3) The deviation angle in the case of the deviated pursuit.

4) The proportionality factor in the case of the proportional navigation.

In some situations, the path traveled by $R_{i+1}$ under the velocity pursuit is not exactly the path traveled by $R_{i}$. There exists an error between $P_{i+1}(t)$ and $P_{i}(t)$ when the path of $R_{0}$ is curved and the distance traveled by $R_{i+1}$ is smaller than the distance traveled by $R_{i}$. The deviated pursuit and the proportional navigation can be used for the path correction of the following robots. This task is accomplished by choosing the appropriate values of the deviation angle or the navigation constant. For the deviated pursuit, this is illustrated in Fig. 4. Clearly, the velocity pursuit requires $R_{i+1}$ to move on the line of sight with $R_{i}$. This path is different from $P_{i}$. The deviation angle $\alpha_{i+1}$ allows to perform a lag pursuit in order to match $P_{i+1}$ and $P_{i}$. The price to pay is a higher value of $v_{i+1}$ compared to the velocity pursuit. Note that the error is small as $d_{i 0}$ is small, but it becomes more important when $d_{i 0}$ increases. Note also that this error does not affect the tracking process.

The implementation of the method for the three guidance laws strategies requires the knowledge of the linear and the angular velocity of the lead robot and the line of sight angle. This can be accomplished by using continuous measurements. The algorithm can also be implemented using a communication system, where each lead robot communicates information concerning its linear velocity and its orientation angle to its following robot. Moreover, the integration of the three guidance strategies in the same robotic convoy is possible.

\section{SimUlation}

We present examples where the robotic convoy is simulated. The convoy consists of five or six robots. For simplicity, we assume that the velocities, the distances, and the time are without units. We consider different types of motion, such as circular motion, sinusoidal motion, and spiral motion. The influence of the sensors noise in considered in the last example. 


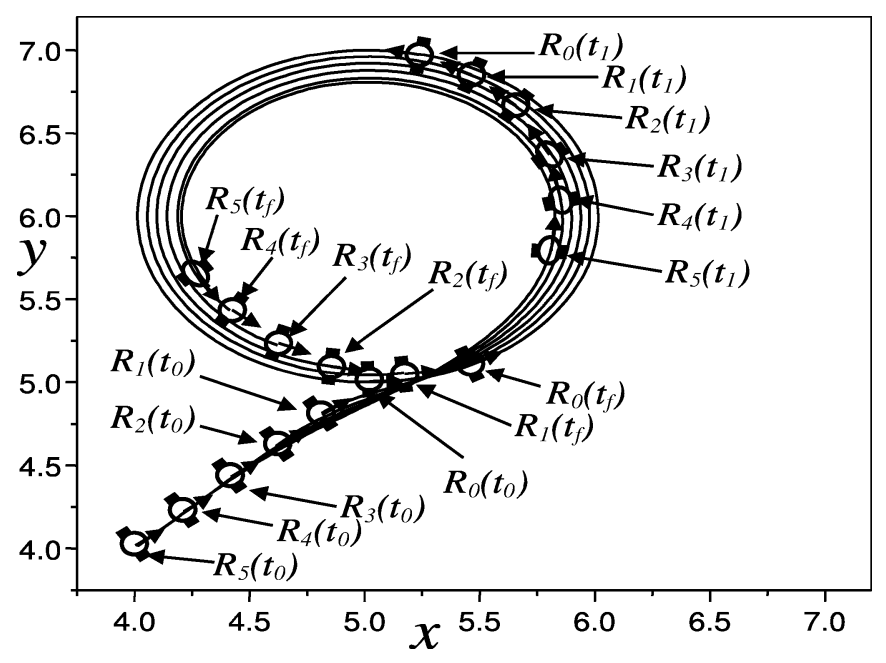

Fig. 5. Path traveled by the convoy, lead robot moving in a circle. The following robots are moving using the velocity pursuit.

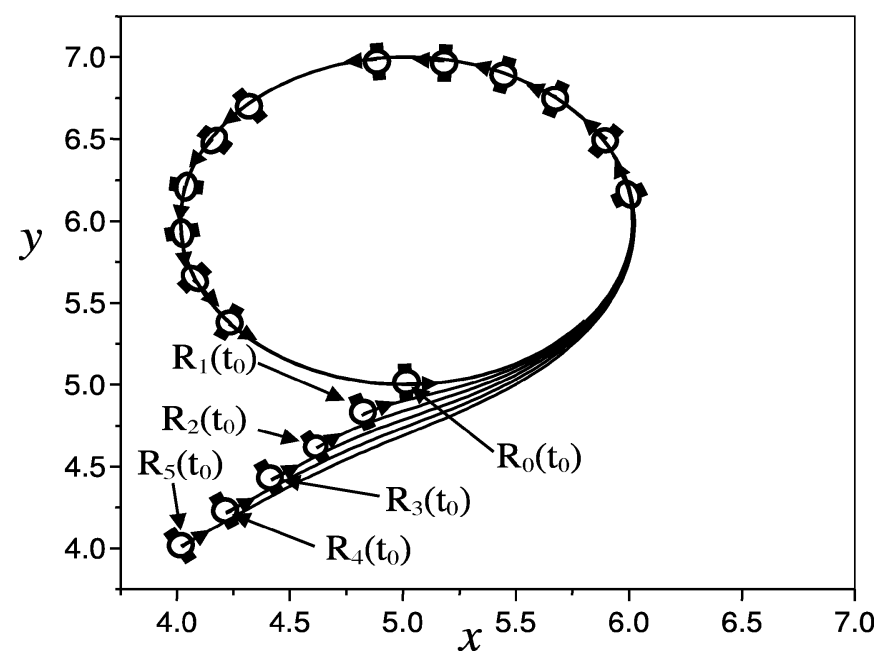

Fig. 6. Path traveled by the convoy, lead robot moving in a circle. The following robots are moving using the deviated pursuit.

Example 1: Lead robot moving in a circle.

Here, the lead robot moves with constant angular velocity and $\theta_{0}(t)=w_{0} t$. There exists a maximum number of robots which can move in a circle with a given radius. We assume that the radius of the circle is large enough so that a specific number of robots can move in the circle. We also assume that $d_{i 0}$ are small enough so that each robot in the circle can point to its lead robot. As we mentioned previously, there exists an error in the path when the lead robot moves in a curved path and the following robots navigate using the velocity pursuit. Our aim in this example is to illustrate the path correction using the deviated pursuit, when the lead robot moves in a circle.

Simulation for this case is illustrated in Figs. 5 and 6, where the following robots navigate using the velocity pursuit and the deviated pursuit, respectively.

After the transient phase, the motion of all following robots becomes circular for all three strategies. However, the following remarks are due.

1) For the velocity pursuit, all robots in the convoy move in a circular motion, however the radius for robot $R_{i+1}$ is

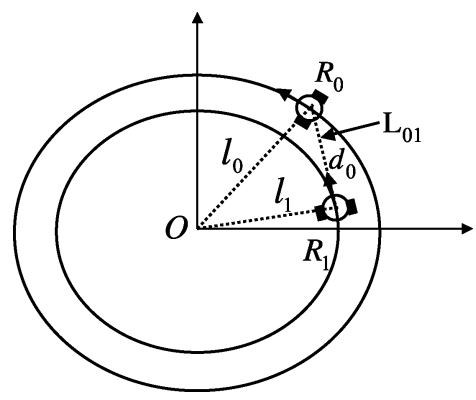

Fig. 7. Error in the path when the lead robot moves in a circle and the following robot is using the velocity pursuit.

slightly smaller than the radius for $R_{i}$, as shown in Fig. 5. The error in the radius depends crucially on the distance $d_{i 0}$. This error becomes very small for small values of $d_{i 0}$.

2) It is possible to correct the path of the following robots by using the deviated pursuit or the proportional navigation. This can be accomplished by acting on the deviation angle or the navigation constant. In this case, it is particularly simple to calculate the deviation angle or the navigation constant so that successive robots move in the same path. The path correction is illustrated in Fig. 6, where the deviation angle is given by $\alpha_{i+1}=0.13 r d(i=0, \ldots, 4)$.

The error in the radius between two successive robots can be determined as follows.

Consider a convoy of two robots, a lead robot $R_{0}$ and a following robot $R_{1}, d_{0}$ is the distance between the robots. At the end of transient period, the lead robot moves in a circle of radius $c_{0}$ and the following robot moves in a circle of radius $c_{1}$. Let us take the origin of the reference frame $O$ at the center of the two circles as shown in Fig. 7. According to the first equation in system (9), the distance between point $O$ and $R_{0}$ (denoted by $l_{0}$ ) and the distance between point $O$ and $R_{1}$ (denoted by $l_{1}$ ) vary as follows:

$$
\begin{aligned}
& \dot{l}_{0}=v_{0} \cos \left(\theta_{0}-\sigma_{0}\right) \\
& \dot{l}_{1}=v_{1} \cos \left(\theta_{1}-\sigma_{1}\right) .
\end{aligned}
$$

Since the robots move in a circle, we have $\dot{l}_{0}=0=\dot{l}_{1}$ and thus $\theta_{0}-\sigma_{0}= \pm \pi / 2$ and $\theta_{1}-\sigma_{1}= \pm \pi / 2$. (Clearly, with this particular choice of point $O$, we have $c_{0}=l_{0}$ and $c_{1}=l_{1}$ ). According to the velocity pursuit, we have $\theta_{2}=\sigma_{01}$, which means that $\sigma_{01}-\sigma_{1}= \pm \pi / 2$, and thus, the line of sight $\mathbf{L}_{01}$ is perpendicular to $\mathbf{l}_{1}$, as shown in Fig. 7. This allows us to write

$$
l_{0}^{2}+d_{0}^{2}=l_{1}^{2} .
$$

Now, in order to see the influence of $d_{0}$, we write $d_{0}$ as a fraction of $l_{1}$, i.e., $d_{0}=l_{1} / m$, and we replace in (73) to get

$$
\begin{aligned}
& l_{0}^{2}=l_{1}^{2}-\frac{l_{1}^{2}}{m^{2}} \\
& l_{0}^{2}=l_{1}^{2}\left(1-\frac{1}{m^{2}}\right) .
\end{aligned}
$$

Equation (74) allows us to express the error between $l_{0}$ and $l_{1}$ as a function of $m$, which represents the ratio between the radius of $R_{0}$ and the distance $\left(R_{0}-R_{1}\right)$. From (74), the error becomes smaller for smaller values of $d_{0}$ (higher values of $m$ ). 


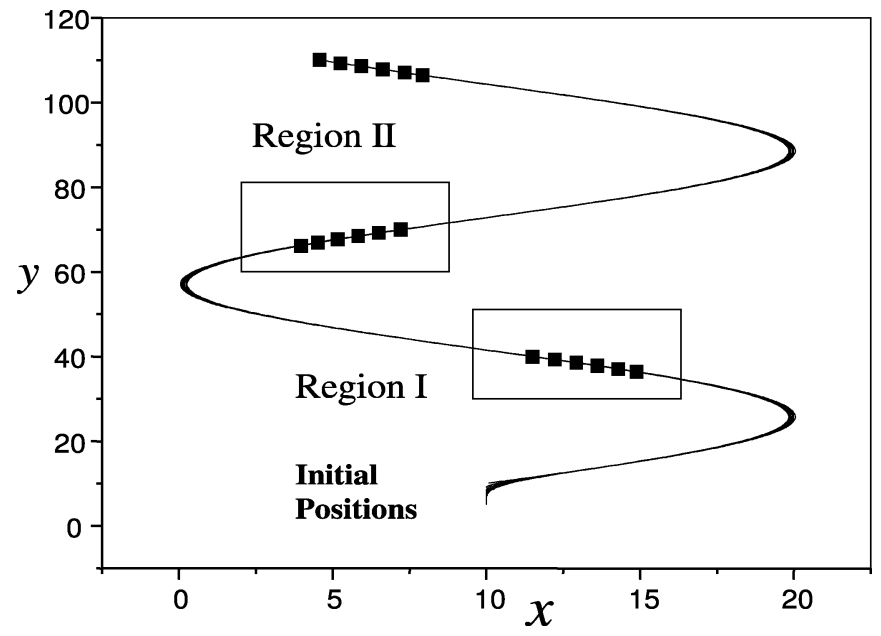

Fig. 8. Robotic convoy moving in a sinusoidal motion. The following robots move according to the velocity pursuit.
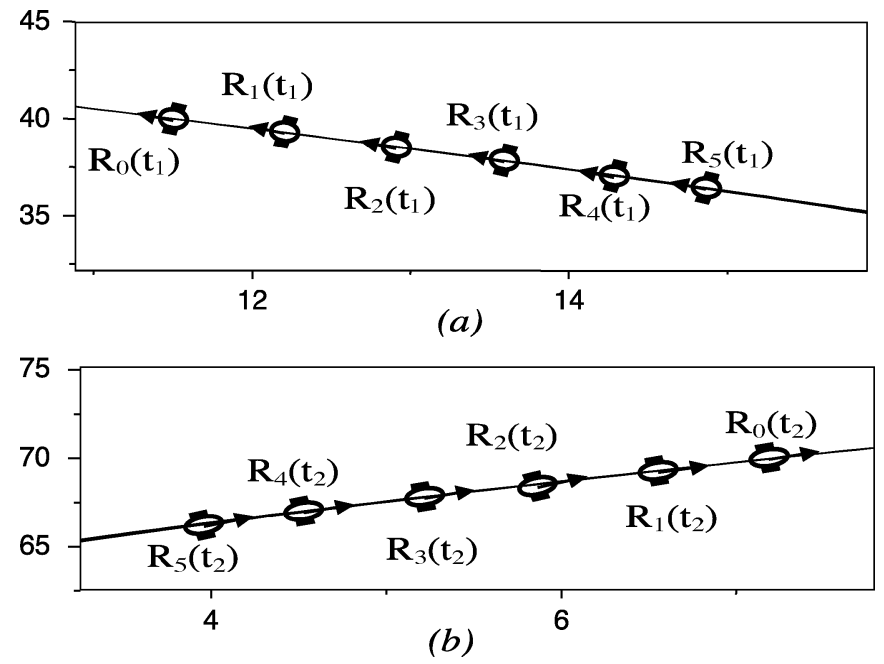

Fig. 9. Enlargements in regions I and II in Fig. 8. (a) Enlargement in region I. (b) Dnlargement in region II.

For example, if $l_{0}=1$, and $d_{0}=0.1$, then $l_{1}=0.9950$, and the error between $c_{0}$ and $c_{1}$ is 0.005 , which is a small error.

It is worth noting that the deviated pursuit can be used also to correct the robots paths, when the robots do not present the same turning characteristics. For example, in the case when $R_{i}$ can perform a turn with a given radius, while $R_{i+1}$ cannot perform the same turn because of the constraint on its turning radius. The appropriate choice of $\alpha_{i+1}$ allows to $R_{i+1}$ to follow $R_{i}$ with some deviation and satisfy the constraint on its turning radius.

Examples 2 and 3 illustrate the case when the lead robot moves with a variable curvature along the path.

Example 2: Lead robot moving in sinusoidal motion.

This scenario is illustrated in Fig. 8. The following robots are using the velocity pursuit. Fig. 9 shows enlargements in regions I and II in Fig. 8. The orientation angle of the robots in the convoy is depicted in Fig. 10. Clearly the orientation angle for $R_{i}(i=1, \ldots, 5)$ is just a delayed version of $\theta_{0}(t)$.

Example 3: Robots moving in spiral motion using the deviated pursuit.

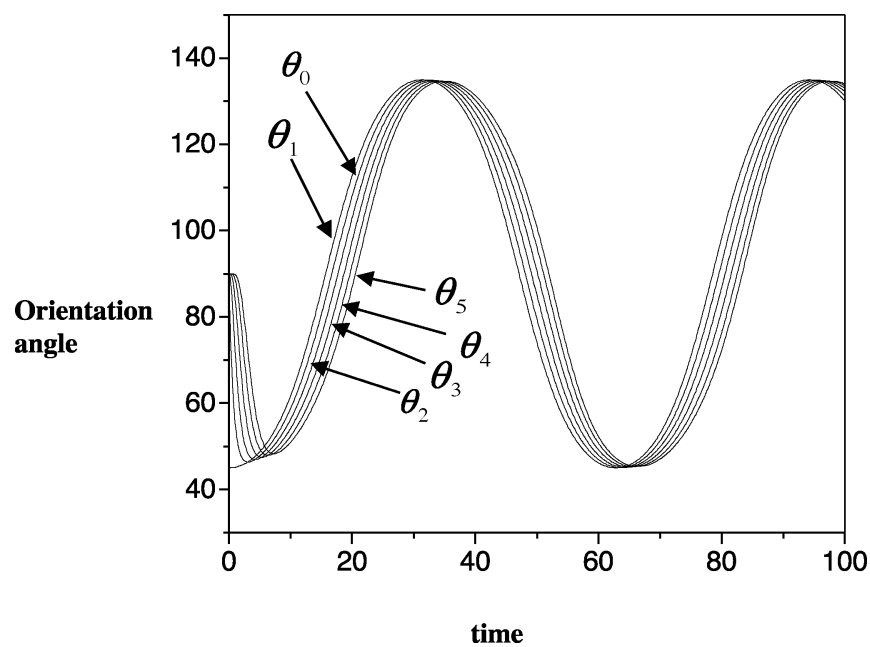

Fig. 10. Orientation angle for the robots in the convoy moving in a sinusoidal motion.

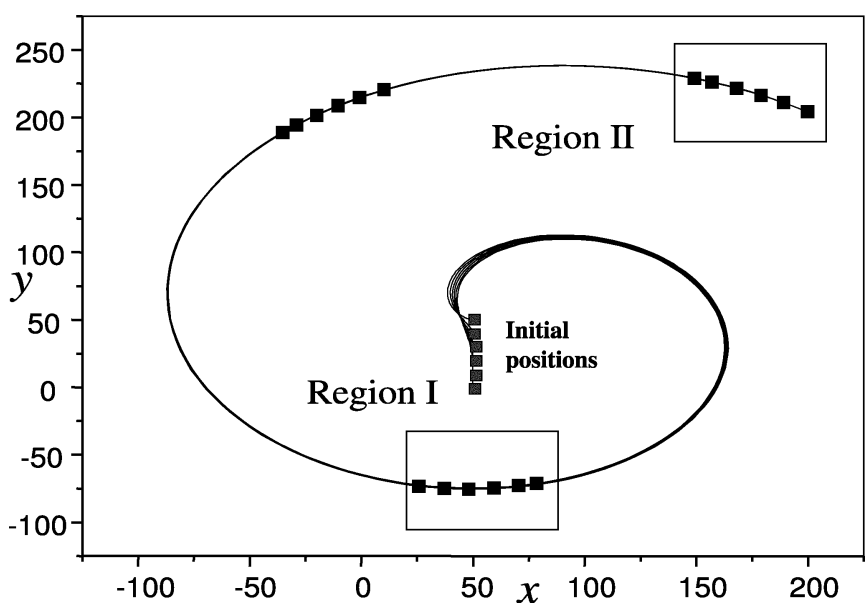

Fig. 11. Robotic convoy moving in a spiral motion. The following robots move according to the deviated pursuit.

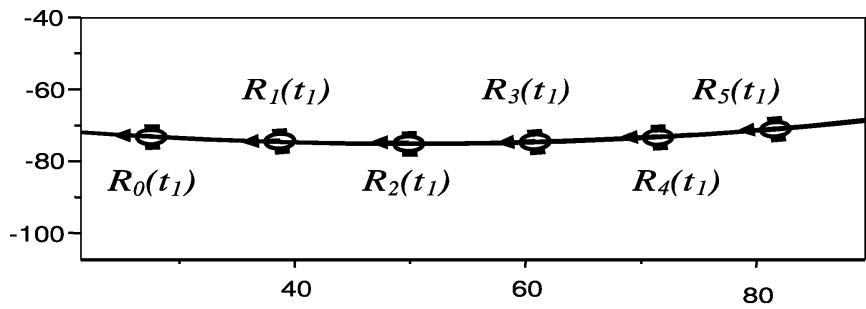

(a)

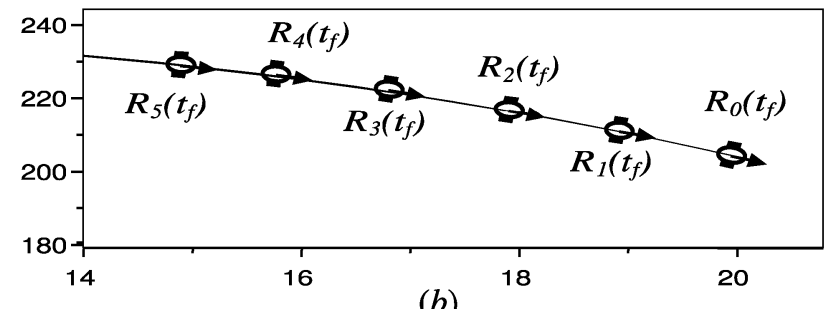

(b)

Fig. 12. Enlargements in regions I and II in Fig. 11. (a) Enlargement in region I. (b) Enlargement in region II. 


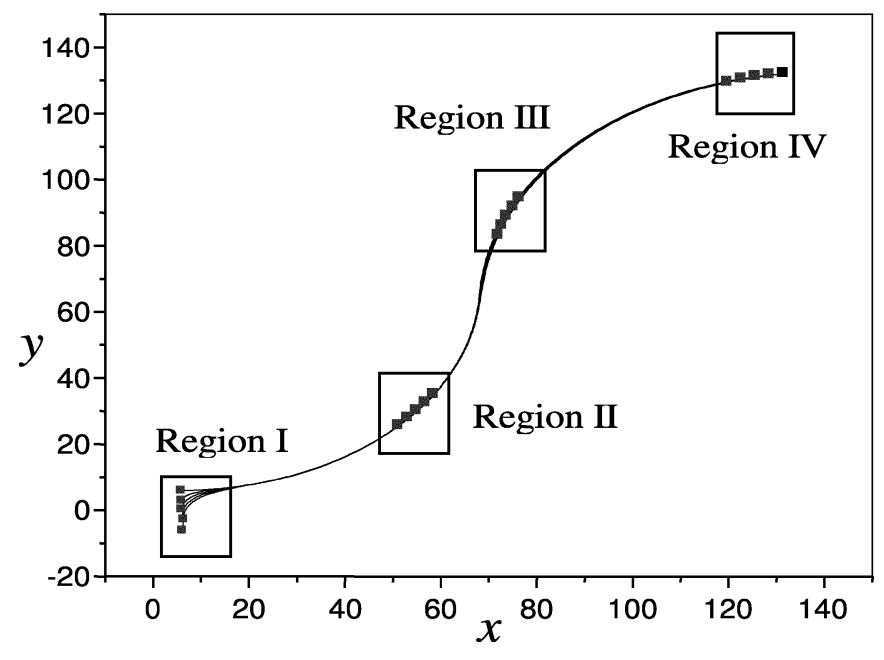

Fig. 13. Path traveled by the convoy using the proportional navigation.

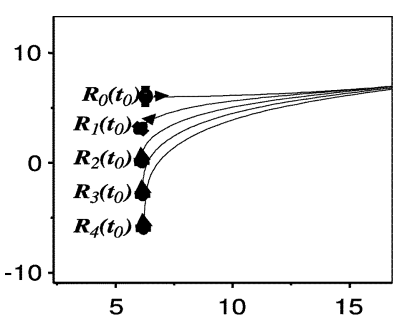

(a)

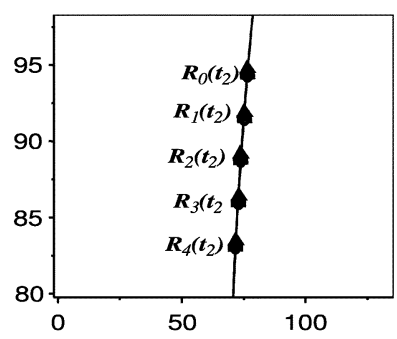

(c)

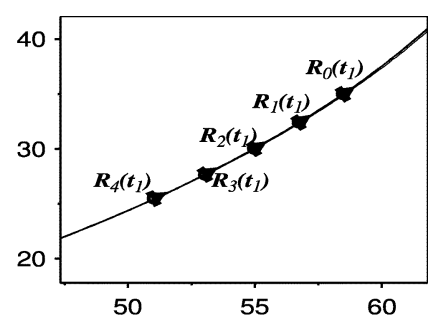

(b)

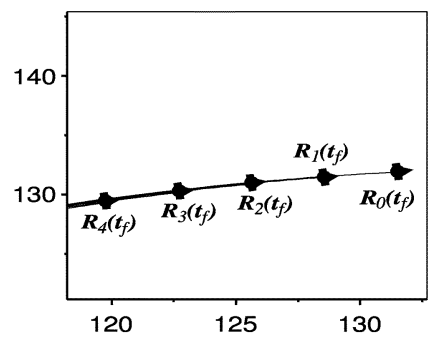

(d)
Fig. 14. Enlargement in regions I through IV in Fig. 13. (a) Enlargement in region I. (b) Enlargement in region II. (c) Enlargement in region III. (d) Enlargement in region IV.

The scenario for this example is shown in Fig. 11, where the following robots use the deviated pursuit. Enlargements in region I and region II in Fig. 11 are shown in Fig. 12.

Example 4: Navigation using the proportional navigation.

This example illustrates the navigation using the proportional navigation. The navigation constant is $K=1.01(M=0.01)$. The convoy consists of five robots, and is illustrated in Fig. 13. Enlargements in regions I-through IV in Fig. 13 are shown in Fig. 14.

Similar to any control or navigation strategy, the navigation strategy based on guidance laws can be affected by sensor noise. This problem is briefly considered in the next example.

Examples 5 and 6 illustrate convoys with a larger number of robots.

Example 5: A relatively complex path.

This example presents a relatively complex path, where the robots move in lines and curves of different radius of curvature. The convoy uses the velocity pursuit. Simulation of this example is shown in Fig. 15 for a convoy of 12 robots. Clearly,

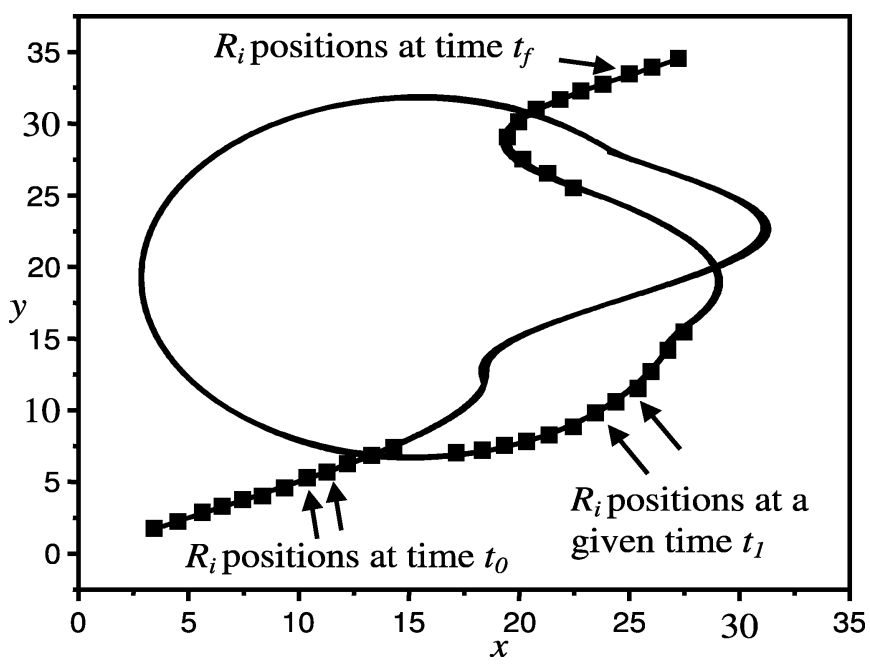

Fig. 15. Robot convoy moving in a relatively complex path.

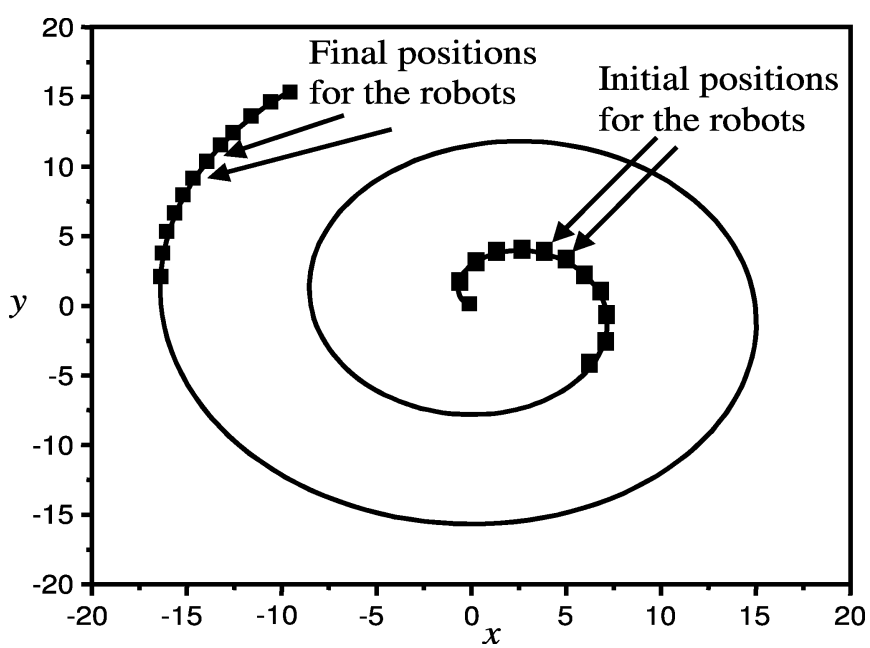

Fig. 16. Robotic convoy moving in a spiral motion. The following robots move according to the velocity pursuit.

the complexity of the trajectory does not affect the control strategies, since complex trajectories can be seen as a combination of simple trajectories.

Example 6: Robots moving in spiral motion using the velocity pursuit.

The convoy moves in a spiral motion similar to example 3 . However, the convoy uses the velocity pursuit instead of the deviated pursuit. Simulation of a convoy of 12 robots for this example is shown in Fig. 16.

Example 7: In this example, our aim is to briefly study the influence of sensor noise on the path of the following robots. Sensor noise can affect all measured quantities, such as the line of sight angle, or the lead robot's orientation angle or linear velocity. We restrict our study to the error due to the measurement of the line of sight angle. The measured value of the line of sight angle between $R_{0}$ and $R_{1}$ is given by

$$
\sigma_{M 01}(t)=\sigma_{01}(t)+n(t) .
$$

We consider two cases. In the first case, $n(t)$ is a random function (as shown in Fig. 17). In the second case, $n(t)$ is proportional to the line of sight angle, we take $n(t)=\sigma_{01}(t) / 3$. Note 


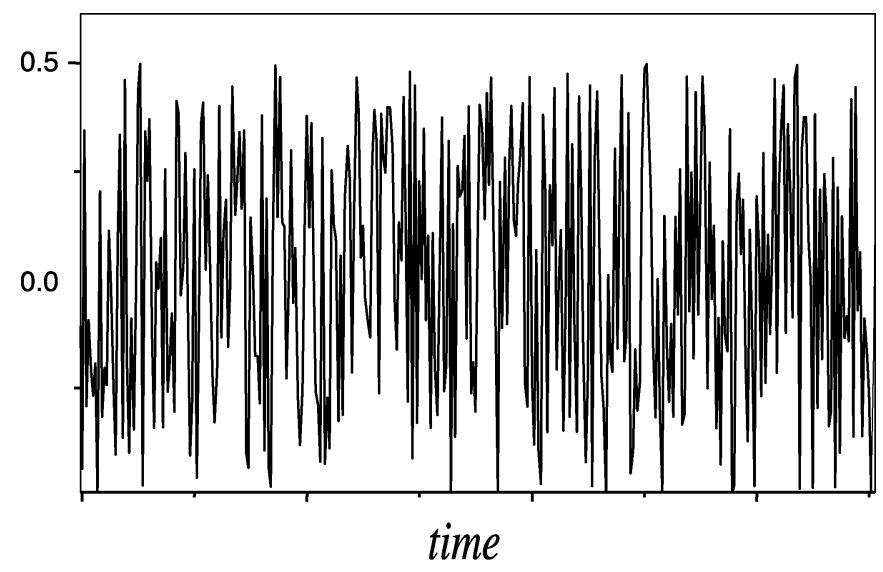

Fig. 17. Random noise affecting the line of sight angle.

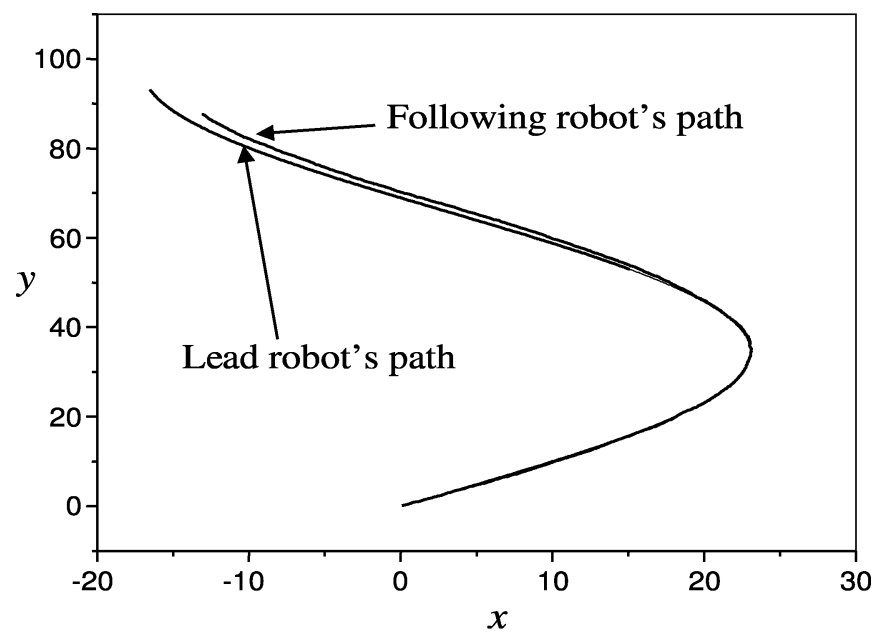

Fig. 18. Path of a two-robot convoy in the presence of random noise.

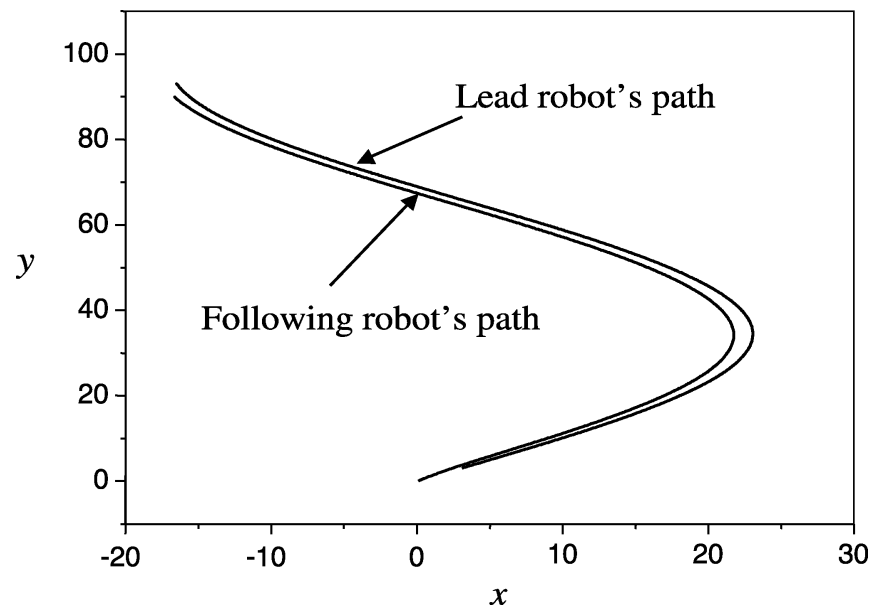

Fig. 19. Path of a two-robot convoy in the presence of proportional noise.

that this error is quite large. Simulation of the convoy for both cases is shown in Figs. 18 and 19. The error in the path can be seen in these figures. However, this error is small when considering the noise level. Different techniques such as filtering can be used to improve this aspect.

\section{CONCLUSION}

The theoretical framework of controlling a convoy of wheeled mobile robots was considered in this paper. The control strategy is derived on guidance laws based on geometrical rules. This formulation of the problem of robotic convoy facilitates modeling of various tracking systems based on sensors or vision. It also facilitates a rigorous analysis of these systems. Three different methods are used and two control laws for each following robot are derived. The control law for the orientation angle is directly derived from the guidance laws equations after elaboration of the kinematics equations between successive robots. The second control law designed for the linear velocity aims to keep the distance between robots constant. Here, the kinematics equations between two successive robots subject to the guidance law are used. Important properties of the method are discussed. Unlike visual servoing methods, which are feature-based methods, the strategies suggested here are model-based, since they explicitly use the kinematics models of the robots in the convoy. Model-based methods may be more accurate for fast and realtime tracking. Unlike vision-based methods, they do not require the processing of the huge amount of data coming from the camera or camera calibration. Both strategies use Kalman filtering to predict and estimate the position/velocity of the lead robot.

It is shown that for all three strategies, each following robot imitates its lead robot, where the angular velocity of each following robots tracks the angular velocity of its lead robot, and the linear velocity of each following robots tracks the linear velocity of its lead robot. Simulation confirms the validity of our approach.

\section{REFERENCES}

[1] S. Feyrer and A. Zell, "Tracking and pursuing persons with a mobile robot," in Proc. Int. Workshop on Recognition, Analysis and Tracking Faces and Gestures in Real-time Systems, Los Alamitos, CA, Sep. 1999, pp. 83-88.

[2] — , "Detection, tracking, and pursuit of humans with an autonomous mobile robot," in Proc. IEEE/RSJ Int. Conf. Intelligent Robots and Systems, Kyonglu, Korea, Oct. 1999, pp. 864-869.

[3] A. Crétual, F. Chaumette, and P. Bouthemy, "Complex object tracking by visual servoing based on 2 d image motion," in Proc. IEEE Int. Conf. Pattern Recognition, Brisbane, Australia, Aug. 1998, pp. 1251-1254.

[4] E. Marchand, P. Bouthemy, F. Chaumette, and V. Moreau, "Robust real-time visual tracking using a 2d-3d model-based approach," in Proc. IEEE Int. Conf. Computer Vision, Keryra, Greece, Sep., p. 1999.

[5] F. Bensalah and F. Chaumette, "Compensation of abrupt motion changes in target tracking by visual servoing," in Proc. IEEE/RSJ Int. Conf. Intelligent Robots and Systems, 1995, pp. 181-187.

[6] L. Hsu and F. Lizarralde, "Robust adaptive visual tracking control: analysis and experiments," in Proc. IEEE Int. Conf. Control Applications, Anchorage, AK, Sep. 2000, pp. 25-27.

[7] F. Chaumette, P. Rives, and B. Espiau, "Positioning of a robot with respect to an object, tracking it and estimating its velocity by visual sevoing," in Proc. IEEE Int. Conf. Robotics and Automation, Palo Alto, CA, Apr. 1991, pp. 2248-2253.

[8] J. Dias, C. Paredes, I. Fonseca, H. Araujo, J. Batista, and A. Almeida, "Simulating pursuit with machine experiments with robots and artificial vision," IEEE Trans. Robot. Autom., vol. 14, no. 1, pp. 1-17, Feb. 1998.

[9] Z. Xiyong and Z. Jing, "Virtual local target method for avoiding local minimum in potential field based robot navigation," J. Zhejiang Univ. Sci., vol. 4, no. 3, pp. 1-17, 2003.

[10] L. Sung-On, C. Young-Jo, H. Myung, Y. Bum-Jae, and O. Sang-Rok, "A stable target-tracking control for unicycle mobile robots," in Proc. IEEE/RSJ Int. Conf. Intelligent Robots and Systems, Takamatsu, Japan, Oct. 2000, pp. 1822-1827. 
[11] M. M. M. Brackstone, "Car-following: a historical review," Transport. Res. F, vol. 2, pp. 181-196, 1999.

[12] H. Yamaguchi and J. Burdick, "Time-varying feedback control for nonholonomic mobile robots forming group formations," in Proc. IEEE Conf. Decision and Control, Tampa, FL, Dec. 1998, pp. 4156-4163.

[13] H. Yamaguchi, T. Arai, and G. Beni, "A distributed control scheme for multiple robotic vehicles to make group formations," Robot. Auton. Syst., vol. 36, pp. 125-147, 2001.

[14] K. C. Cheok, G. Smid, K. Kobayashi, J. Overholt, and P. Lescoe, "A fuzzy logic intelligent control system paradigm for an in-line-of-sight leader-following hmmwv," J. Robot. Syst., vol. 14, no. 6, pp. 407-420, 1997.

[15] R. Holve, P. Protzel, J. Bernasch, and K. Naab, “Adaptive fuzzy control for driver assistance in car-following," in Proc. European Congr. Intelligent Techniques and Soft Computing, Aachen, Germany, Aug. 1995, pp. 1149-1153.

[16] G. Dudek, M. Jenkin, E. Milios, and D. Wilkes, "Experiments in sensing and communication for robot convoy navigation," in Proc. IEEE/RSJ Int. Conf. Intelligent Robots and Systems, Los Alamitos, CA, Aug. 1995, pp. 268-273.

[17] C. Canudas-deWit and A. Ndoudi-Likoho, "Nonlinear control for a convoy-like vehicle," Automatica, vol. 36, pp. 457-462, 2000.

[18] H. Schneiderman, M. Nashman, A. Wavering, and R. Lumia, "Visionbased robotic convoy driving," Mach. Vis. Applicat., vol. 8, pp. 359-364, 1995.

[19] M. Schwarzinger, T. Zielke, D. Noll, M. Brauckmand, and W. von Seelen, "Vision-based car-following: detection, tracking, and identification," in Proc. Intelligent Vehicles Symp., Detroit, MI, Jun. 1992, pp. 24-29.

[20] T. Zielke, M. Brauchkmann, and W. von Seelen, "Cartrack: computer vision-based car following," in Proc. IEEE Workshop on Applications of Computer Vision, Detroit, MI, Nov. 1992, pp. 156-163.

[21] - "Intensity and edge-based symmetry detection with application to car-following," Image Understanding, vol. 58, no. 2, pp. 177-190, 1993.

[22] N. Shneydor, Missile Guidance and Pursuit: Kinematics, Dynamics and Control. Chichester, U.K.: Harwood, 1998.

[23] M. McBeath, D. Shaffer, and M. Kaiser, "How baseball outfielders determine where to run to catch fly balls," Science, vol. 268, pp. 569-573, 1995.

[24] A. Bruckstein, "Why the ant trails look so straight and nice," The Mathematical Intelligencer, vol. 15, no. 2, pp. 59-62, 1993.

[25] S. Rumeliotis, P. Pirjanian, and M. J. Mataric, "Ant-inspired navigation in unknown environments," in Proc. Autonomous Agents, Barcelona, Spain, Jun. 2000, pp. 25-26.

[26] D. Lambrinos, "Navigation in desert ants: the robotics solutions," Robotica, vol. 21, no. 4, pp. 407-426, 2003.

[27] U. Nehmzow, "Animal and robot navigation," Robot. Auton. Syst., vol. 15, pp. 71-81, 1995.

[28] D. Lambrinos, R. Moller, R. P. T. Labhart, and R. Wehner, "A mobile robot employing insect strategies for navigation," Robot. Auton. Syst., vol. 30, pp. 39-64, 2000.

[29] M. Srinivasan, J. Chahl, K. Weber, S. Venkatesh, M. Nagle, and S. Zhang, "Robotic navigation inspired by principles of insect vision," Robot. Auton. Syst., vol. 26, pp. 203-216, 1999.
[30] H. Piccardo and G. Honderd, "A new real-time algorithm for robotic manipulator path planning to enable the grasoing of moving targets: Some design aspects," in Proc. Int. Conf. Control, Edinburgh, U.K., Mar. 1991, pp. 795-800.

[31] M. Mehrandezh, M. Sela, R. Fenton, and B. Benhabib, "Proportional navigation guidance for robotics interception of moving objects," $J$. Robot. Syst., vol. 17, no. 6, pp. 321-340, 2000.

[32] M. Mehrandezh, N. M. Sela, R. G. Fenton, and B. Benhabib, "Robotic interception of moving objects using an augmented ideal proportional navigation guidance techniques," IEEE Trans. Syst., Man. Cybern. A Syst. Humans, vol. 30, no. 3, pp. 238-250, May 2000.

[33] M. Aicardi, G. Casalino, A. Bicchi, and A. Balestrino, "Closed loop steering of unicycle-like vehicles via lyapunov techniques," IEEE Robot. Autom. Mag., vol. 2, no. 1, pp. 27-35, Mar. 1995.

[34] J. Yang and J. Kim, "Sliding mode control for trajectory of nonholonomic wheeled mobile robots," IEEE Trans. Robot. Autom., vol. 15, no. 3, pp. 578-586, Jun. 1999.

[35] M. Guelman, "A qualitative study of proportional navigation," IEEE Trans. Aerosp. Electron. Syst., vol. AES-7, no. 4, pp. 637-643, 1971.

[36] C. D. Yang and C. Yang, "A unified approach to proportional navigation," IEEE Trans. Aerosp. Electron. Syst., vol. 33, no. 2, pp. 557-567, Apr. 1997.

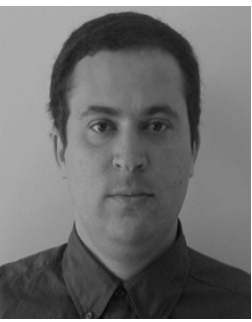

Fethi Belkhouche (M'01) received the B.S. degree in electrical engineering in 1997 and the M.S. degree in electronic physics in July 2001, both from the University of Tlemcen, Algeria. He is currently pursuing the Ph.D. degree in electrical engineering in the Department of Electrical Engineering and Computer Science, Tulane University, New Orleans, LA.

His research interests include guidance theory, robot navigation, robot cooperation and formation control, and linearization methods.

Mr. Belkhouche is a member of the AIAA.

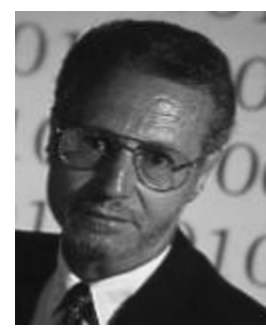

Boumediene Belkhouche (S'82-M' 82 ) is Professor of Electrical Engineering and Computer Science at Tulane University, New Orleans, LA. His areas of research include motion planning for autonomous mobile robots and object-oriented modeling. 